\title{
Respiratory Modulation of Spontaneous Subthreshold Synaptic Activity in Olfactory Bulb Granule Cells Recorded in Awake, Head-Fixed Mice
}

\author{
Isaac A. Youngstrom and Ben W. Strowbridge \\ Department of Neurosciences, Case Western Reserve University, Cleveland, Ohio 44106
}

\begin{abstract}
Although the firing patterns of principal neurons in the olfactory bulb are known to be modulated strongly by respiration even under basal conditions, less is known about whether inhibitory local circuit activity in the olfactory bulb (OB) is modulated phasically. The diverse phase preferences of principal neurons in the $\mathrm{OB}$ and olfactory cortex that innervate granule cells (GCs) may interfere and prevent robust respiratory coupling, as suggested by recent findings. Using whole-cell recording, we examined the spontaneous, subthreshold membrane potential of GCs in the OBs of awake head-fixed mice. We found that, during periods of basal respiration, the synaptic input to GCs was strongly phase modulated, leading to a phase preference in the average, cycle-normalized membrane potential. Subthreshold phase tuning was heterogeneous in both mitral and tufted cells (MTCs) and GCs but relatively constant within each GC during periods of increased respiratory frequency. The timing of individual EPSPs in GC recordings also was phase modulated with the phase preference imparted by large-amplitude EPSPs, with fast kinetics often matching the phase tuning of the average membrane potential. These results suggest that activity in a subset of excitatory afferents to GCs, presumably including cortical feedback projections and other sources of large-amplitude unitary EPSPs, function to provide a timing signal linked to respiration. The phase preference we find in the membrane potential may provide a mechanism to dynamically modulate recurrent and lateral dendrodendritic inhibition of MTCs and to selective engage a subpopulation of interneurons based on the alignment of their phase tuning relative to sensory-driven MTC discharges.
\end{abstract}

Key words: head-fixed mice; interneurons; intracellular recording; olfactory bulb; patch-clamp recording; synaptic potentials

\section{Introduction}

Olfactory information en route to the primary olfactory regions is transformed from a spatiotemporal pattern of glomerular activation by olfactory sensory neurons (OSNs) responding to odorants into the action potentials (APs) of mitral and tufted cells (MTCs), the principal output neurons in the olfactory bulb (OB; Rubin and Katz, 1999; Wachowiak and Cohen, 2001). Both odor-evoked and spontaneous MTC firing exhibit respiratory patterning reflecting the odor-transducing and mechanoreceptive properties of OSNs (Grosmaitre et al., 2007; Carey and Wachowiak, 2011; Cenier et al., 2013). Activity in granule cells (GCs), the most common inhibitory interneuron subtype in the $\mathrm{OB}$, can influence olfactory sensory perception (Abraham et al., 2010), presumably by modulating MTC firing patterns.

Understanding how activity in inhibitory GCs is related to the output of the $\mathrm{OB}$ is a technically difficult question to assess because

Received Jan. 23, 2015; revised April 21, 2015; accepted April 27, 2015.

Author contributions: I.A.Y. and B.W.S. designed research; I.A.Y. performed research; I.A.Y. and B.W.S. analyzed data; I.A.Y. and B.W.S. wrote the paper.

This study was supported by National Institutes of Health Grant R01-DC04285 (B.W.S.). We thank Loren Schmidt and Todd Pressler for helpful discussions related to this project and Dan Wesson for constructive comments on this manuscript.

The authors declare no competing financial interests.

Correspondence should be addressed to Dr. Ben W. Strowbridge, Department of Neurosciences, Case Western Reserve University School of Medicine, 10900 Euclid Avenue, Cleveland, 0H 44106. E-mail: bens@case.edu.

DOI:10.1523/JNEUROSCI.0311-15.2015

Copyright $\odot 2015$ the authors $\quad 0270-6474 / 15 / 358758-10 \$ 15.00 / 0$ both suprathreshold and subthreshold depolarizations in these interneurons can modulate GABA release (Isaacson and Strowbridge, 1998). Phasic modulation of GC membrane potential with the respiratory cycle might function to facilitate dendrodendritic inhibition by varying the probability of dendrodendritic inhibition of MTCs, reflecting transient periods at the depolarizing peaks of the oscillation when the tonic blockade of NMDARs will be minimal. Because both sensory input to the $\mathrm{OB}$ and the firing patterns of MTCs are highly rhythmic (Onoda and Mori, 1980; Grosmaitre et al., 2007; Carey and Wachowiak, 2011; Cenier et al., 2013), determining whether the local circuits that drive GCs preserves presynaptic oscillatory and respiratory phase tuning information. Although some studies have begun to address firing patterns of GCs in the awake state in mammals (Cazakoff et al., 2014), whether the subthreshold synaptic input to GCs is phase modulated with respect to a simultaneously recording respiratory signal is not known. Although the strong respiratory modulation of MTC activity could obviously provide a source of phasemodulated input to GCs, respiratory phase preference is heterogeneous among MTCs (Buonviso et al., 1992; Fukunaga et al., 2012) and piriform cortical neurons (Gire et al., 2013), and destructive interference among different presynaptic inputs may limit or even occlude phase modulation in GCs. Indirect support for the interference model comes from the limited phase modulation observed in GC spiking activity observed during basal conditions (Cazakoff et al., 2014). However, the extracellular spiking 
measurements used in that study would not have detected more robust modulation of subthreshold activity in GCs that could provide a cellular mechanism that phase aligns a subset of GCs spikes.

We performed whole-cell patch-clamp intracellular recordings in vivo in awake, head-fixed mice to examine the subthreshold activity of GCs during basal conditions, when MTC spiking exhibits strong respiratory coupling. We found strong phase modulation in both the subthreshold average membrane potential and the timing of individual EPSPs that were coupled to respiration. GC subthreshold activity, like MTC spike patterns, exhibited diverse phase relationships with the respiratory rhythm, with the largest phase modulation occurring in the timing of large-amplitude EPSPs with rapid kinetics.

\section{Materials and Methods}

In vivo recordings. Wild-type C57BL/6 mice (P23-P35, either sex; Harlan Laboratories) initially underwent a surgical procedure $(100 \mathrm{mg} / \mathrm{kg}$ ketamine and $10 \mathrm{mg} / \mathrm{kg}$ xylazine, i.p., anesthesia) to mount a small titanium plate $(9 \times 25 \mathrm{~mm})$ with two bolt holes on the dorsal surface of the skull using Metabond adhesive (Parkell; for details, see Youngstrom and Strowbridge, 2012). Two days after surgery, mice were positioned near the apex of a levitated 20.3-cm-diameter Styrofoam ball using two 4-40 metal bolts that mounted the head plate to the steel superstructure surrounding the treadmill during conditioning and recording sessions. Mice could sit or walk on the treadmill or groom themselves while head restrained. We conducted three habituation sessions (each 30 min duration) in which mice were head restrained over the spherical treadmill. Motion of the treadmill was detected using an optical mouse (MX-518; Logitech) connected to a netbook computer as described previously (Youngstrom and Strowbridge, 2012).

Mice then underwent a second surgical procedure to create a small craniotomy over the dorsal $\mathrm{OB}(0.5-1.0 \mathrm{~mm}$ diameter; $4.5 \mathrm{~mm}$ rostral, $1.0 \mathrm{~mm}$ lateral to bregma) before the first recording session $(100 \mathrm{mg} / \mathrm{kg}$ ketamine and $5 \mathrm{mg} / \mathrm{kg}$ xylazine, i.p., anesthesia). The craniotomy was irrigated using warmed $\left(40^{\circ} \mathrm{C}\right)$ artificial CSF [in mM: $124 \mathrm{NaCl}, 3 \mathrm{KCl}$, $1.23 \mathrm{NaH}_{2} \mathrm{PO}_{4}, 1.2 \mathrm{MgSO}_{4}, 26 \mathrm{NaHCO}_{3}, 10$ dextrose, and $2.5 \mathrm{CaCl}_{2}$ (equilibrated with $95 \% \mathrm{O}_{2} / 5 \% \mathrm{CO}_{2}$ )]. Except when noted, all compounds used in this study were obtained from Sigma. Patch-clamp electrodes $[1.2 \mathrm{~mm}$ outer diameter, $0.96 \mathrm{~mm}$ inner diameter borosilicate tubing containing a filament (WPI); typical resistance, 6-8 $\mathrm{M} \Omega$ ] were advanced to within $250 \mu \mathrm{m}$ of the pial surface using a motorized manipulator (MP-285; Sutter Instruments) while applying strong positive pressure $(75-90 \mathrm{kPa})$ on the electrode tip. Pipettes were filled with an internal solution that contained the following (in $\mathrm{mM}$ ): $140 \mathrm{~K}$-methylsulfate (MP Biochemicals), $4 \mathrm{NaCl}, 10$ HEPES, 0.2 EGTA, 4 MgATP, $0.3 \mathrm{Na}_{3} \mathrm{GTP}$, and 10 phosphocreatine, $\mathrm{pH} 7.3$ ( $\sim 290 \mathrm{mOsm})$. Once advanced to near the mitral cell layer (MCL), the positive pressure applied to the electrode was reduced to $1.4 \mathrm{kPa}$, and intracellular recordings were attempted using blind patch-clamp methods (Blanton et al., 1989) and an Axopatch 1D amplifier (Molecular Devices). In some experiments, Alexa Fluor-594 (100 $\mu \mathrm{M}$; Invitrogen) was added to the intracellular solution, and we attempted to recover filled neurons after brain fixation with $4 \%$ paraformaldehyde using an Axioskop2 fluorescence microscope (Zeiss). However, the large positive pressures necessary to reliably form patch-clamp recordings and the low yield of successful recordings over multiple penetrations in the same subject precluded unambiguous visualization of recorded neurons. The laminar location of recovered neurons was determined by comparison with landmarks (the glomerular and MCLs) visible using transmitted light microscopy or by examining tissue autofluorescence. Reported voltages are not corrected for the liquid junction potential (approximately $-10 \mathrm{mV}$ ). All recording sessions were conducted on the same day as the craniotomy surgery (range, P24-P36). Subjects recovered from anesthesia within 1-2 h; all subjects had regained their righting reflex and were ambulating before the first attempt at recording. All experiments were approved by the Case Western Reserve University Animal Care and Use Committee.
Electrophysiology and data analysis. Intracellular recordings were lowpass filtered at $5 \mathrm{kHz}$ and acquired simultaneously with treadmill velocity signals and respiration as measured by a thermocouple external to the naris (model TT-K; Omega Engineering) at $10 \mathrm{kHz}$ (ITC-18 interface; InstruTech) using custom VB.NET software. Movement was calculated as the sum of the two absolute velocities acquired. Depth of recording was measured as the distance traveled from the dura by the manipulator guiding the electrode.

AP characteristics were measured using custom-written software in MATLAB (MathWorks). AP threshold was detected using an automated procedure that identified the time immediately before AP response when the second derivative of the membrane potential reached $10 \%$ of its maximal value during the AP upstroke. AP amplitude was measured as the difference between peak and threshold for the first three APs of a step response. AP half-width was measured as the time between the threshold and the peak. Input resistance was measured as the voltage change in response to a $100 \mathrm{pA}$ hyperpolarizing current step. Thermocouple signals were low-pass filtered at $100 \mathrm{~Hz}$, and minima, marking the transition from cooling to warming, were used as fiducial markers of each respiratory cycle. We limited this study to respiratory cycles $>200 \mathrm{~ms}(<5 \mathrm{~Hz}$ instantaneous frequency) because previous work found less reliable phase modulation of MTC spiking with respiratory cycles above this frequency (Carey and Wachowiak, 2011), and the signal-to-noise ratio for detecting transitions between respiratory cycles was optimal during epochs of slower breathing. Restricting the analysis to respiratory cycles with duration $>200 \mathrm{~ms}$ ( $<5 \mathrm{~Hz}$ instantaneous frequency) eliminated $\sim 30 \%$ cycles from the dataset. The recorded membrane potential from each cycle was normalized to the length of the respiratory cycle by dividing it into 720 points, with each point of the normalized membrane potential being the mean of 720 bins, with bin width ranging from 0.2778 to $0.6944 \mathrm{~ms} / \mathrm{bin}$. Vectors representing the phase relationship between membrane potentials and the respiratory cycle were made using each point of the cycle normalized, membrane potential as the magnitude of the vector components, and taking the arc-tangent of the sum of the vector components, as described previously (Goldberg and Brown, 1969; Buonviso et al., 1992). For comparison with the peak amplitude of the mean, phase-aligned membrane potentials, each respiratory cycle vector was shuffled before averaging, and this was repeated 1000 times to produce a population of surrogate means. To compare vector magnitudes with chance, we shuffled each respiratory cycle vector to make a population of 1000 surrogate vectors and then compared the magnitude of the mean vector of the population with the magnitude of the mean vector of the real data. Event detection was performed using custom-written MATLAB software used in previous studies (Hyde and Strowbridge, 2012; Schmidt and Strowbridge, 2014). In brief, an automated algorithm detected continuous clusters of time points when the derivative of the membrane potential exceeded a threshold and was reset by a subsequent cluster of time points with derivatives below a second threshold (e.g., an "up-only" filter). Vectors describing the relationship between postsynaptic events and respiration were composed in a manner similar to the method published previously for spike-time respiratory coupling (Buonviso et al., 1992), i.e., each bin of the respiratory cycle with an event start time was considered a 1 and all other points of the vector as a 0 , before taking the arc-tangent sum of the vector components. We calculated the phase modulation of each cell as measured by the difference between the three bins surrounding the maximum and the three bins surrounding the minimum of the histogram of phase positions of EPSPs, normalized by the total number of events. Data were analyzed using MATLAB 2012a, including the Circular Statistics toolbox (Berens, 2009). Other statistical tests were performed using Origin 8.5 (OriginLab). All data are presented as mean \pm SEM except when noted. Statistical significance was determined using Student's $t$ test unless specified otherwise.

\section{Results}

We recorded intracellularly from 62 OB neurons in awake, headfixed mice operating a levitated spherical treadmill using blind whole-cell patch-clamp methods (Blanton et al., 1989). The firing patterns and AP shape of most recorded neurons were con- 


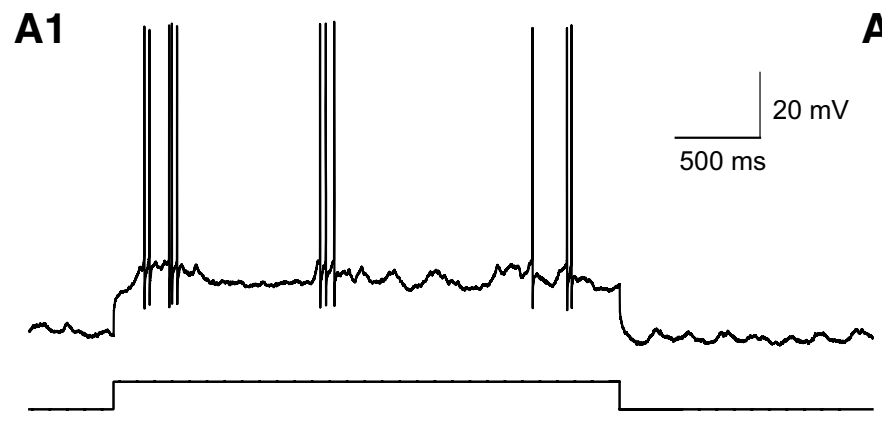

A2
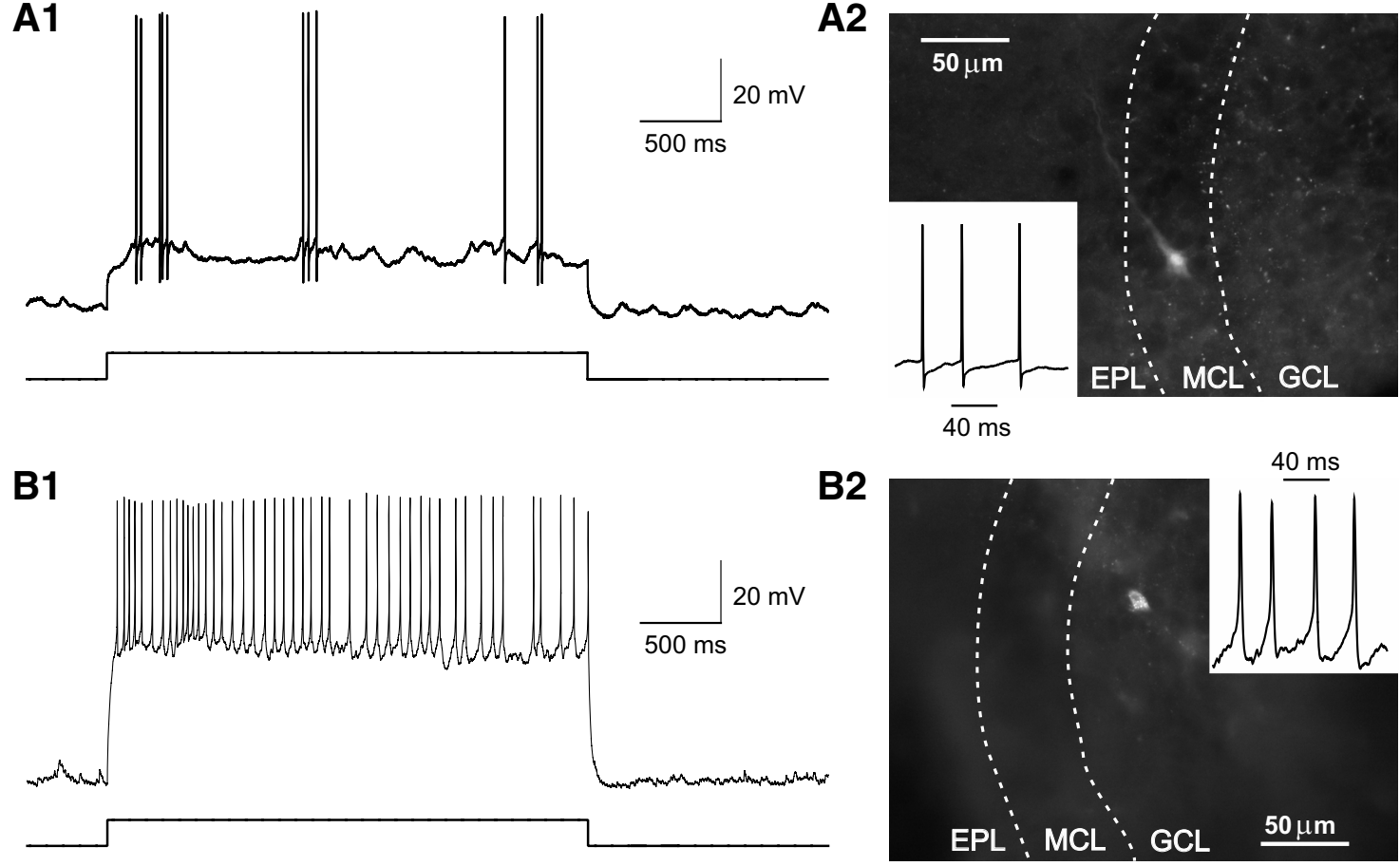

B2

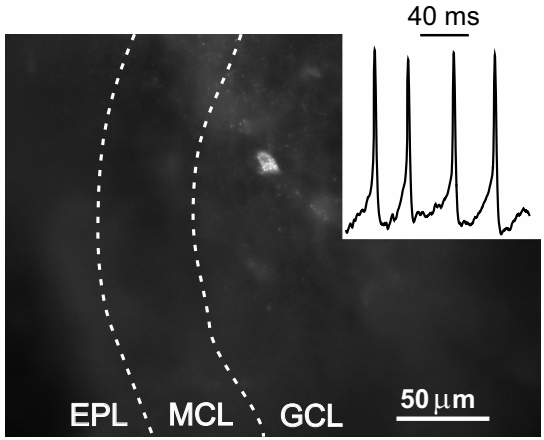

C

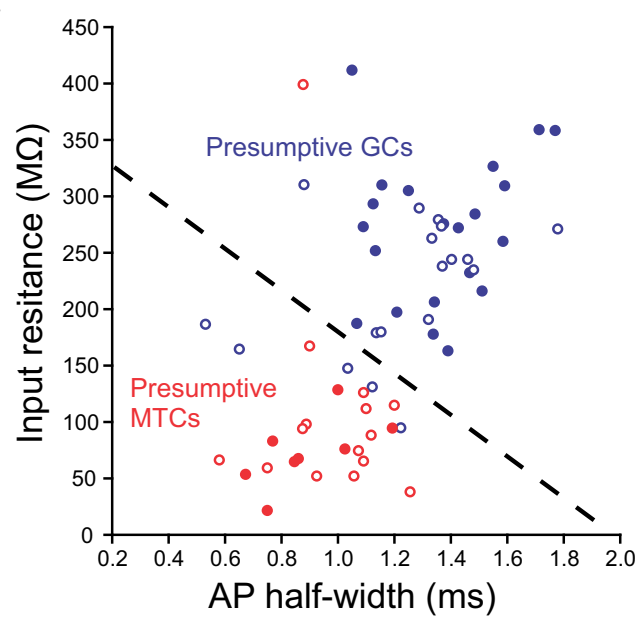

D

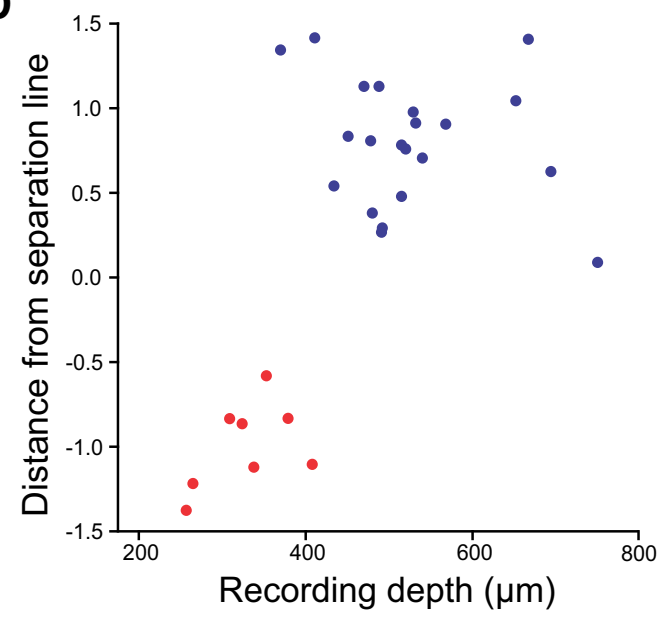

Figure 1. Intracellular recordings from MTCs and GCs in awake, head-fixed mice. $\boldsymbol{A}$ 1, Response to a depolarizing current step in a presumed MTC recorded at a depth of $408 \boldsymbol{\mu m}$ has an intermittent firing pattern typical of mitral cells. A2, Photomicrograph of a different mitral cell filled with Alexa Fluor-594 through the intracellular recording electrode with a cell body in the MCL (cell recorded at a depth of $327 \mu \mathrm{m})$. Dashed lines indicate borders between the EPL, the MCL, and the GCL. B1, Response to depolarizing current step in a presumed GC recorded at a depth of $520 \mu \mathrm{m}$. B2, Intracellularly filled $\mathrm{GC}$ recorded at a depth of $516 \mu \mathrm{m}$. Inset, Enlargement of the depolarizing step response of the filled GC in B2. C, Plot of input resistance versus AP half-width for 62 OB neurons recorded from 56 head-fixed mice. Symbol colors represent presumptive GCs (blue; mean recording depth, $508 \pm 15 \mu \mathrm{m}$ ) and MTCs (red; recording depth, $324 \pm 12 \mu \mathrm{m}$; significantly different from $\mathrm{GCs} ; p<5 \times 10^{-12}$, $t$ test) based on visual inspection of responses to depolarizing current steps. Filled symbols represent neurons used in subsequent phase coupling analysis (recordings capturing $>100$ respiratory cycles). Dashed separation line determined by LDA based on entire dataset (filled and open symbols). $\boldsymbol{D}$, Plot of recording depth versus distance from LDA separation line in $\boldsymbol{C}$ for the subset of neurons used for phase coupling analysis. Filled blue symbols are presumptive $\mathrm{GCs}(n=21)$, and red symbols represent presumptive MTCs ( $n=8$ ). Open blue symbols below the separation line, and open red symbols above the line were misclassified by the LDA procedure.

sistent with previous reports of either MTCs (Isaacson and Strowbridge, 1998; Margrie et al., 2001) or GCs (Margrie and Schaefer, 2003; Balu et al., 2007). MTC-like discharges were composed of APs that overshot $0 \mathrm{mV}$ (18 of 23 putative MTCs; Fig. $1 A 1)$ that were generated within intermittent clusters. Figure $1 A 2$ illustrates a partially recovered mitral cell that responded to depolarizing current steps with APs typical of MTCs (inset). In contrast, presumptive GCs generated smaller-amplitude, longerduration APs than MTCs (AP half-width at half-maximal ampli- tude, $0.93 \pm 0.04$ vs $1.30 \pm 0.04 ; p<10^{-6}, t$ test) and typically discharged tonically (Fig. 1B1). Visualized GCs $(n=13$; Fig. 1B2) had small-diameter $(<15 \mu \mathrm{m})$ cell bodies located within the GC layer (GCL) and fired tonically (Fig. 1B2, inset). Presumptive MTCs and GCs could be classified using linear discriminant analysis (LDA) based on standard intrinsic properties (Fig. 1C). Of the $62 \mathrm{OB}$ neurons recorded in this study, only six cells appeared to be misclassified based on visual inspection of responses to depolarizing current steps using the two-factor LDA shown in 
A1

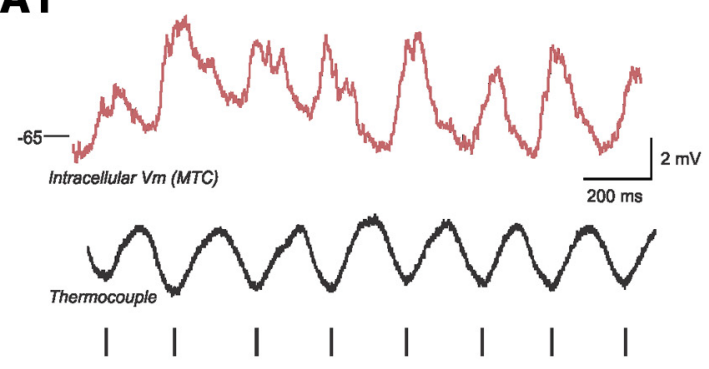

A2

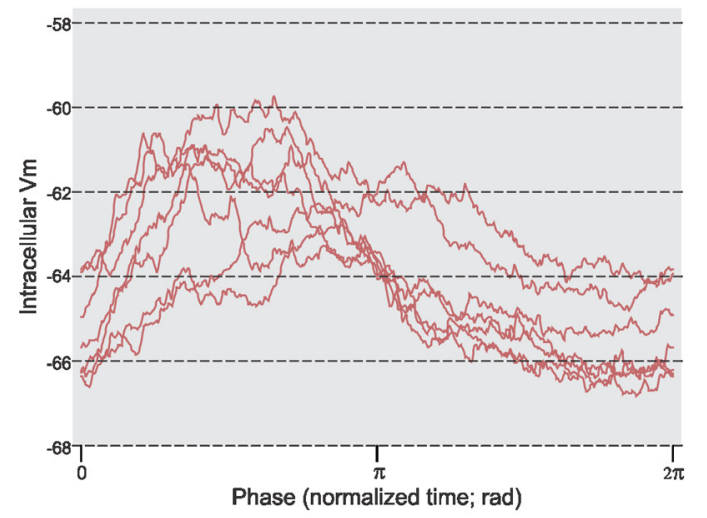

A3

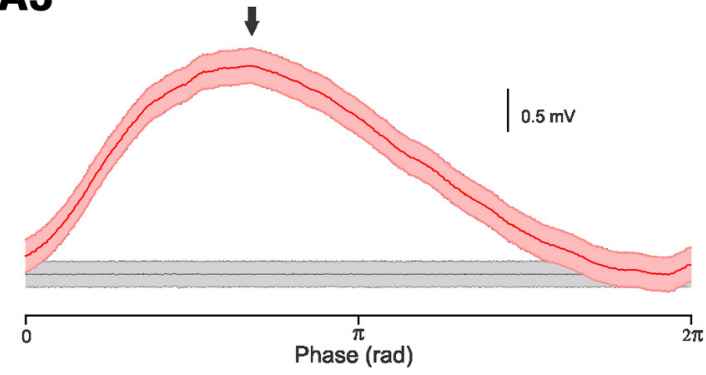

B1

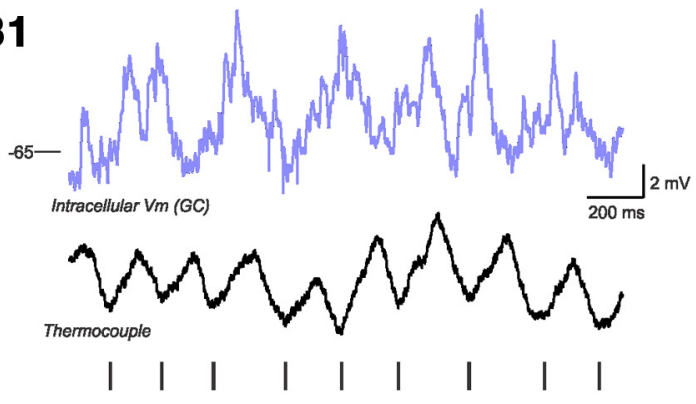

B2

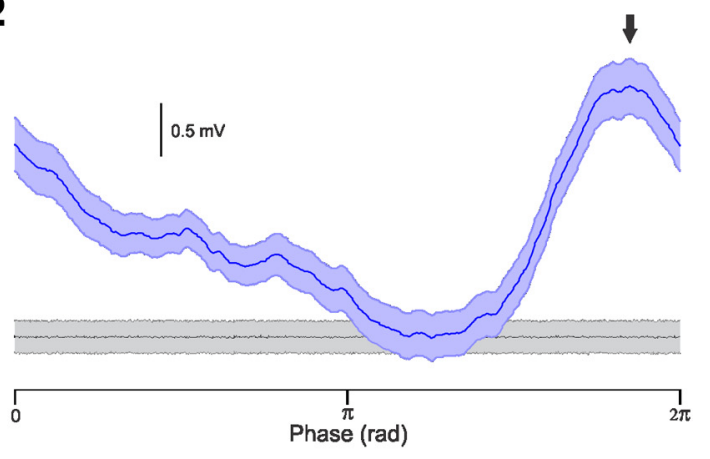

C

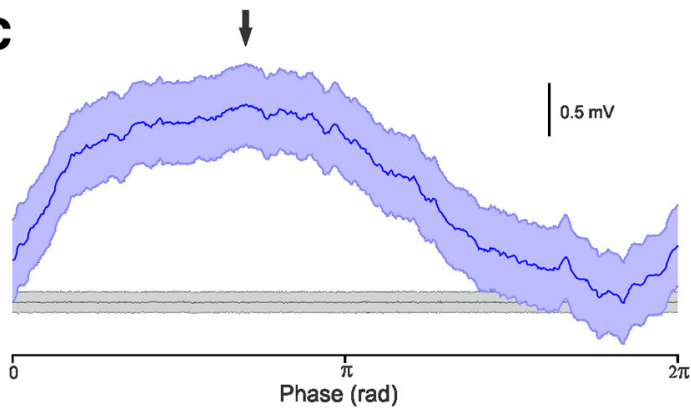

Figure 2. Phase modulation of MTC and GC membrane potentials. $\boldsymbol{A}$ 1, The intracellular membrane potential of an MTC (red) above the concurrently recorded respiration (black), as measured by a thermocouple placed directly in front of the external naris of the animal. Vertical lines indicate the minima of the thermocouple recording, used to distinguish respiratory cycles. Upward deflections in the thermocouple signal indicate exhalation, as measured by the warming of the thermocouple. $\mathbf{A 2}$, Membrane potential recording from $\boldsymbol{A} \mathbf{1}$, split into respiratory cycles and normalized to the period of each respiratory cycle to generate phase-aligned, membrane potential activity. $\mathbf{A}$, The mean of all phase-aligned respiratory cycles between 2 and $5 \mathrm{~Hz}$ from the cell in $\boldsymbol{A} \mathbf{1}(n=325$ cycles; shared regions represents 1 SEM). The black line is the mean of 1000 averages of surrogate data with bin order shuffled, with SE shown in the gray shaded region. The arrow indicates the phase of peak depolarization of the mean membrane potential (2.1 rad). B1, The intracellular membrane potential of a GC (blue) with the simultaneously recorded respiratory signal (black). Vertical lines indicate the respiratory trace minima. $\boldsymbol{B 2}$, The mean, phase-aligned membrane potential of the cell in $\boldsymbol{A} \mathbf{1}$ is shown in blue ( $n=302$ cycles), with SE in the shaded region, overlaying the mean of the shuffled, surrogate data (black), with SE in the gray shaded region. The arrow indicates the phase of peak depolarization of the mean membrane potential (5.9 rad). $C$, The mean, phase-aligned membrane potential of a second $\mathrm{GC}$ (blue, $n=332$ cycles), with SE shown in the shaded region, and the mean of the surrogate data shown in black, with SE in the shaded region. The phase tuning of this GC (arrow at $2.2 \mathrm{rad}$ ) was different from the GC shown in $\boldsymbol{B}$.

Figure $1 C$. This automatic classification method based on intrinsic properties also correlated well with recording depth (Fig. 1D), suggesting that our intracellular recording procedure reliably sampled from both principal cell and interneuron OB populations in awake, head-fixed mice. Because we cannot exclude that some rare but large tonic-firing interneurons located in the GCL (Blanes and Golgi cells; Pressler and Strowbridge, 2006; Pressler et al., 2013) were also recorded, we use the abbreviation "GC" to include both GCs and GCL layer interneurons.

We next focused on a subset of eight MTCs and 21 GCs recordings in which we were able to extensively sample subthreshold synaptic activity over at least 100 respiratory cycles. All 29 neurons used to assay respiratory coupling were correctly categorized by the two-factor LDA presented above (Fig. 1C, filled symbols). The intrinsic properties of the two classes of recorded neurons resemble values for input resistance $(78.6 \pm 12 \mathrm{M} \Omega, n=$
8 MTCs; and $265 \pm 15 \mathrm{M} \Omega, n=21 \mathrm{GCs})$ and AP half-width $(0.884 \pm 0.063 \mathrm{~ms}, n=8 \mathrm{MTCs}$; and $1.34 \pm 0.049 \mathrm{~ms}, n=21$ GCs) reported in the literature for these cell types. In both brain slice (Chen and Shepherd, 1997; Lowe, 2002; Pressler and Strowbridge, 2006; Pressler et al., 2007) and in vivo studies (Margrie and Schaefer, 2003; Fukunaga et al., 2012), MTCs display lower input resistances $(\sim 50-100 \mathrm{M} \Omega)$ than GCL interneurons $(>200$ $M \Omega$ ). These studies also consistently report that MTCs have narrower APs than GCs. Consistent with previous intracellular recording studies in awake mice (Fukunaga et al., 2012), we find that the membrane potential of MTCs was well coupled to the basal respiratory rhythm (Fig. 2A). The membrane potential during individual respiratory cycles was dominated by smallamplitude EPSPs (Fig. 2A2). When the time axis was normalized to determine the respiratory phase relationship of the intracellular response ("phase warping"; Goldberg and Brown, 1969; 
Buonviso et al., 1992; Shusterman et al., 2011), we observed obvious phase tuning patterns in both superimposed responses during individual cycles and the average membrane potential (mean \pm SEM shown in Fig. $2 A 3$ over 302 cycles). The phase modulation of MTC membrane potential was much greater than observed by averaging the membrane potential after shuffling bin order in the same neuron (Fig. 2A3, gray trace).

Although phase modulation of subthreshold MTC responses have been described previously (Fukunaga et al., 2012; Phillips et al., 2012), it is not known whether the synaptic input to GCs in awake mammals is coupled as directly to the respiratory cycle. GCs receive monosynaptic excitatory input from MTCs and, therefore, could reflect the strong phase modulation present in these principal cells. However, GCs also receive large-amplitude excitatory inputs from piriform cortical neurons (Balu et al., 2007; Boyd et al., 2012). Phase tuning also varies among principal cells in the OB (Buonviso et al., 1992; Fukunaga et al., 2012) and cortical neurons (Gire et al., 2013), leading to the possibility that diverse inputs across the large dendritic arborization of GCs could "average out" the strong phase preference of individual presynaptic MTCs or cortical neurons.

GC intracellular activity was typically nosier than MTC recordings (mean membrane potential variance, $40 \pm 6.8 \mathrm{mV}^{2}$ for GCs vs $11 \pm 1.9 \mathrm{mV}^{2}$ for MTCs; $p<0.002$, $t$ test, $\left.n=27\right)$. GC responses also typically showed more cycle-to-cycle variation in the magnitude of the respiratory-related depolarization (Fig. 2B1). Despite the larger membrane potential noise and variability, GCs typically displayed clear phase modulation, as shown in the example average membrane potential trace in Figure 2B2. As with MTCs, the magnitude of phase modulation of GC membrane potentials often far exceeded the magnitude expected by chance (Fig. 2B2, gray trace). GCs also exhibited variation in their phase tuning, as illustrated by the example mean GC responses shown in Figure 2B2 (maximal membrane potential modulation at 5.9 rad; arrow) and Figure $2 C$ (2.2 rad, in a different GC).

Over the population of eight MTCs and 21 GCs analyzed for phase coupling, $\sim 80 \%$ had statistically significant phase modulation of subthreshold intracellular responses (larger magnitudes than the 95th percentile of the shuffled, cycle-normalized membrane potential in the same neurons; 6 of 8 MTCs and 18 of 21 GCs). The population average amplitude of phase modulation also was significantly greater in both MTCs and GCs compared with shuffled control responses $(p<0.05, n=8$ and $p<$ $10^{-6}, n=21$, respectively, paired $t$ test; Fig. $3 A$ ). The average membrane potential of both MTCs and GCs also had statistically significant phase modulation when assayed using vector magnitudes calculated from histograms that included all phase-aligned membrane potential time bins $(p<0.03$ vs shuffled responses, $n=8$ MTCs; $p<5 \times 10^{-7}, n=21$ GCs; Fig. $3 B$; for details, see Fukunaga et al., 2012). Both MTCs and GCs had similar tuning widths (full-width at half-maximal amplitude, $3.0 \pm 0.33 \mathrm{rad}$ for MTCs and $3.1 \pm 0.20 \mathrm{rad}$ for GCs; $p>0.05, t$ test, $n=24)$, and the peak phase preference spanned a similar range (from 0.99 to 3.7 rad over six MTCs with statistically significant phase modulation and from 1.2 to 5.9 rad for $18 \mathrm{GCs}$; Fig. 3C). The degree of phase modulation estimated by the vector magnitude representations (Fig. 3C) also did not differ significantly between MTCs and GCs $(p>0.05, t$ test, $n=24)$. These results demonstrate that the magnitude and variation in tuning of intracellular membrane potential phase modulation was similar in both principal cells and GCs.

Phase tuning in the mean membrane potential in both MTCs and GCs was relatively stable after changes in respiration rate and during epochs of spontaneous motion on the spherical treadmill. We analyzed modulation of phase tuning by dividing respiratory cycles based on instantaneous frequency $(2-3.5$ and $3.5-5 \mathrm{~Hz})$ or cycles that occurred with average treadmill motion $>0.1 \mathrm{~cm} / \mathrm{s}$ or during quasi-stationary periods $(<0.1 \mathrm{~cm} / \mathrm{s}$ treadmill motion). As shown in the example plots in Figure $4, A$ and $B$, from the same GC, phase tuning was maintained under these different conditions, in both the magnitude of phase-related modulation of the membrane potential and phase tuning. Over the population of 18 GCs with statistically significant phase modulation, the angular difference between the phase directions during low and high respiration rates and between motion and no motion conditions were significantly less than the variation in phase direction we observed between different GCs $\left(p<5 \times 10^{-5}\right.$ and $p<2 \times$ $10^{-6}$, respectively; Fig. $4 C$ ). The average phase preference calculated during each condition also was not statistically different from the preference calculated from surrogate data with randomized condition (high or low respiration frequencies, motion or no motion) labels ( $p>0.05$; Fig. $4 C$ ). There also was no significant difference between the phase preference in high versus low respiratory rates or between motion and no motion conditions in the six MTCs with statistically significant membrane potential phase modulation ( $p>0.05$; Fig. $4 D$ ). These results suggest that phase modulation during basal respiration is robust and relatively constant over the time course of our intracellular recordings (typically $10-20 \mathrm{~min}$ ).

We next sought to define the types of excitatory synaptic input that determine phase preference in GCs. When phase-aligned membrane potentials were compared, GCs have significantly greater variance than MTCs $\left(27 \pm 6.7\right.$ vs $11 \pm 1.9 \mathrm{mV}^{2}, p<$ $0.002, t$ test), consistent with increase in overall (without phase warping) membrane potential variance described above. The increased variance in GCs could arise from variability in the timing of individual synaptic events, variability in EPSP amplitude, or a combination of these factors. (We held GCs in most recordings near $E_{\mathrm{Cl}-}$ minimize the contribution of $\mathrm{GABA}_{\mathrm{A}}$ receptormediated IPSPs to membrane potential fluctuations.) However, GC and MTC membrane potential variance did not differ when compared after "amplitude normalization" in which the peak-topeak amplitude of each cycle was normalized to 1 in every cell $\left(0.15 \pm 0.007\right.$ vs $0.13 \pm 0.01 \mathrm{mV}^{2}, p>0.05, t$ test $)$. The large decrease in variance observed in GCs after amplitude normalization suggests that the increased variability of GC membrane potential coupling to the respiratory cycle arises primarily from variability in EPSP amplitude rather than variability in the timing of synaptic events.

We next determined the timing of individual EPSPs relative to the respiratory cycle. In most GCs, both EPSP amplitude and kinetics were highly variable, with occasional EPSPs as large as 15 $\mathrm{mV}$ and a wide distribution of $20-80 \%$ rise times from $<1$ to 10 $\mathrm{ms}$ (Fig. $5 A$, insets). Although the distribution of EPSP timing across the respiratory cycle was non-uniform in most GCs with membrane potential phase coupling ( 16 of 18 GCs with $p<0.05$ against a uniform distribution hypothesis, Rayleigh's test), many GCs exhibited only minimal obvious phase coupling of EPSP times in the histograms such as from the example GC shown in Figure 5. This finding raises the possibility that phase coupling of the GC membrane potential may arise predominately from a subset of excitatory inputs.

We tested that hypothesis by examining separately largeamplitude ( $>4 \mathrm{mV}$; Fig. $5 B$, orange bars) EPSPs with fast kinetics $(<3 \mathrm{~ms}$ rise times; Fig. $5 \mathrm{C})$ and small-amplitude $(<4 \mathrm{mV}$; Fig. $5 B$, green bars) EPSPs with slow kinetics ( $>3$ ms rise times; Fig. 

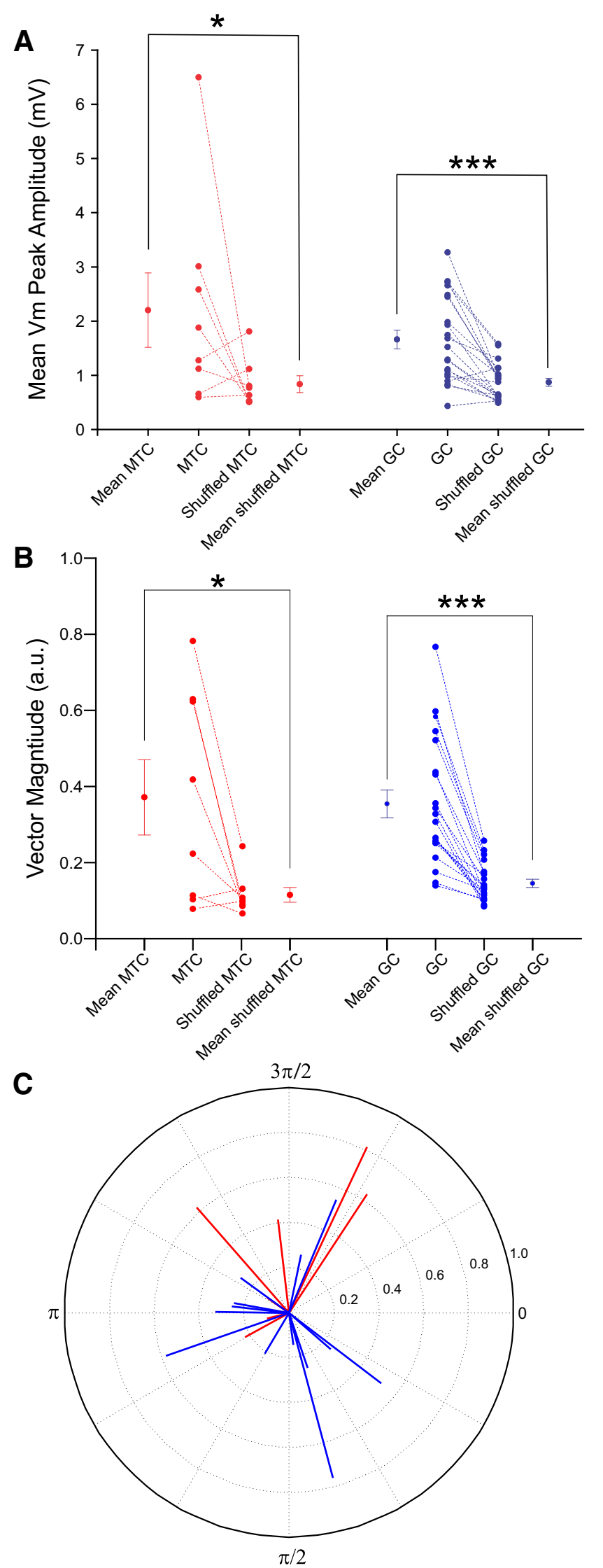

Figure 3. Summary of phase MTC and $\mathrm{GC}$ membrane potential phase modulation. $A$, Comparison of peak amplitudes of the mean, phase-aligned membrane potentials of MTCs (red) and GCs (blue) compared with the 95th percentile of peak amplitudes of their respective shuffled datasets. The means and SEs of the group and of the grouped surrogate data are on either side
$5 C)$. These two groups accounted for $9.5 \pm 1.4$ and $24 \pm 2.8 \%$ of the overall population of spontaneous EPSPs in the $18 \mathrm{GCs}$ with statistically significant membrane potential phase coupling. In an example GC with robust membrane potential phase coupling (Fig. 5D), only the average rate of large-amplitude EPSPs with fast kinetics showed robust phase modulation (Fig. 5E, orange histogram; modulation amplitude, $19.8 \%$ ), whereas the average rate of both small, slow EPSPs (green histogram) and the average rate of all EPSPs (gray histogram) showed little phase modulation (6.7 and 2.7\% modulation, respectively). The phase preference evident in the population of large, fast EPSPs (2.3 rad; orange vector in the polar plot shown in Fig. $5 F$ ) was more similar to the phase preference computed from the mean membrane potential (2.9 rad; Fig. 5F, black vector) than the vector computed from slow, small-amplitude EPSPs (5.2 rad). The slight phase advance of increase in large, fast EPSP (assayed by onset time) relative to the mean membrane potential response is consistent with modulation of the membrane potential arising, at least in part, from temporal summation large-amplitude EPSPs with fast rise times.

The overall population of $18 \mathrm{GCs}$ with significant membrane potential coupling followed the same pattern with statistically greater phase modulation in the subset of large-amplitude EPSPs with fast kinetics than either the subpopulation of smallamplitude EPSPs with slow kinetics $(p<0.005)$ or the phase modulation calculated from all EPSPs $\left(p<5 \times 10^{-4}\right.$; Fig. $\left.5 G\right)$. Consistent with the peaked distribution of large, fast EPSPs, we found that the kurtosis of the distribution of large, fast EPSP phases $(-0.68 \pm 0.1)$ was significantly more positive than either the kurtosis of small, slow EPSP times $(-1.16 \pm 0.02 ; p<2 \times$ $\left.10^{-5}\right)$ or all EPSPs times $\left(-1.15 \pm 0.01 ; p<2 \times 10^{-4}, t\right.$ test $)$.

Next, we estimated the phase tuning from different EPSP subpopulations and asked how well phase preference calculated from different ensembles of individual synaptic inputs matched the average membrane potential phase tuning. Over the population of 18 GCs with statistically significant phase modulation of the membrane potential, population response vectors computed from fast EPSPs spanned the largest range of phase preferences (4.2 rad; Fig. 6A), closely matching the range of vector directions computed from phase, aligned membrane potentials (4.7 rad; $p>0.05$, Cramer-Von Mises test). Phase preference vectors derived from all EPSPs and small, slow EPSPs spanned a smaller range of directions (both $3.8 \mathrm{rad}$ ). The magnitudes of response vectors computed from all EPSPs and the two EPSP subgroups were all significantly larger than shuffled event vectors $(p<5 \times$ $10^{-8}, p<5 \times 10^{-10}$, and $p<10^{-9}$, respectively, paired $t$ test; Fig. $6 B)$, suggesting that at least some phase preference information is distributed throughout the overall population of EPSPs. However, the magnitude of the response vectors computed from large, fast EPSPs was significantly larger than vectors derived from small, slow EPSPs ( $p<0.01$, paired $t$ test). The magnitude of the large, fast EPSP phase preference vectors was not significantly different from response vectors computed from the average membrane potential $(p>0.05)$, whereas both the vectors computed from all EPSPs and the small, slow subpopulation of EPSPs were significantly smaller than the vectors representing

$\leftarrow$

of the individual means. $\boldsymbol{B}$, Comparison of vector magnitudes estimated from mean membrane potentials for the same groups presented in $\boldsymbol{A}$. $\boldsymbol{C}$, Polar plot representing the angles and magnitudes of the vectors created from phase-aligned membrane potentials in MTCs (red; $n=6$ ) and GCs (blue; $n=18$ ) with statistically significant membrane potential phase modulation (peak phase modulation $>95$ percentile of shuffled responses). ${ }^{*} p<0.05 ;{ }^{* * *} p<0.001$. 
A

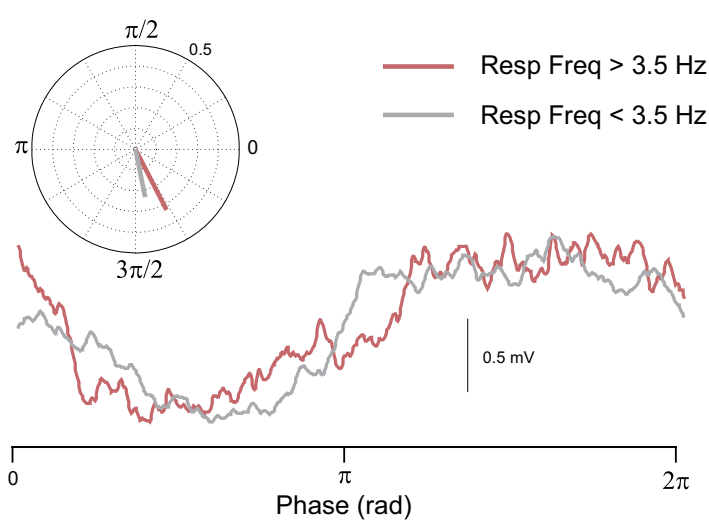

B
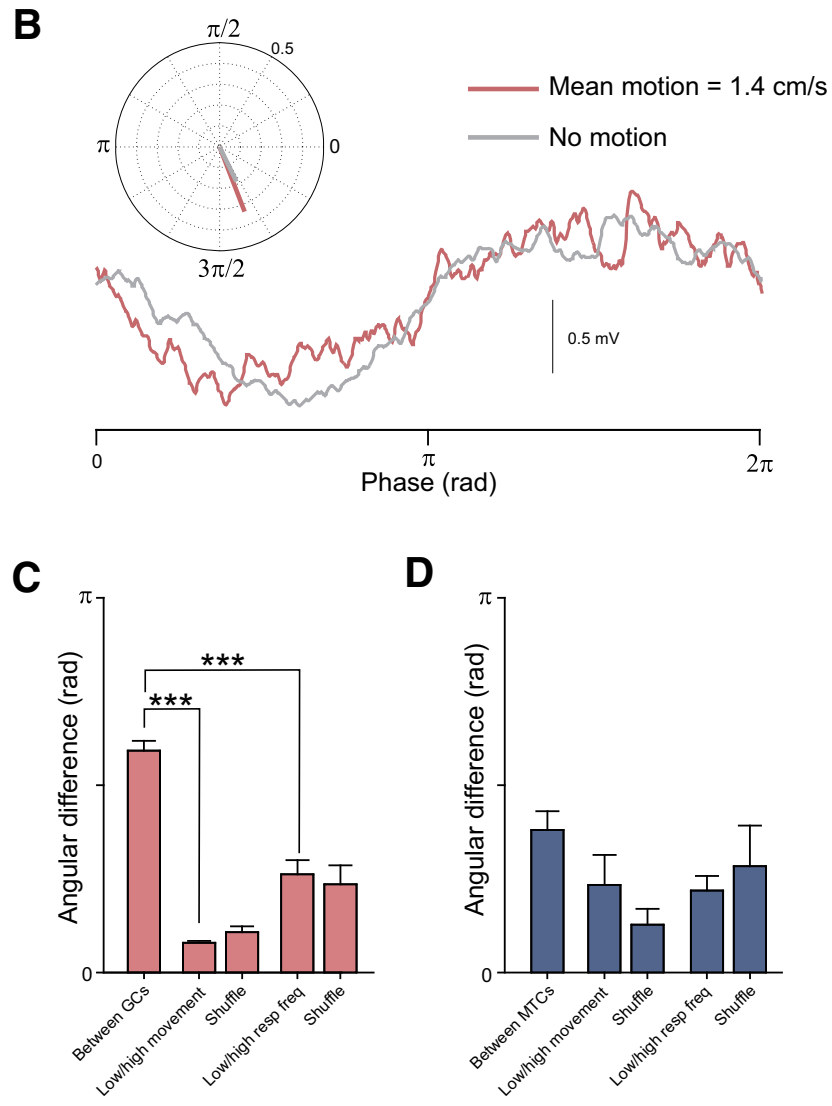

Figure 4. Little modulation of membrane potential phase tuning after changes in respiratory frequency or with locomotion. $A$, The mean, phase-aligned membrane potential from a GC during the high respiratory frequency condition (red trace; respiratory frequencies between 3.5 and $5 \mathrm{~Hz}$; mean respiratory frequency, 4.01; $n=215$ cycles) and during the low respiratory frequency condition (gray; between 2 and $3.5 \mathrm{~Hz}$; mean frequency, 3.14; $n=140$ cycles). Inset, Polar plot with vectors representing the angle and magnitude of the phase relationship of the phase-aligned mean membrane potential during low (gray) and high (high) respiratory frequency conditions. $\boldsymbol{B}$, The mean, phase-aligned membrane potential from the same $\mathrm{GC}$ during the high motion condition (red; $>0.1 \mathrm{~cm} / \mathrm{s}$; mean motion, $1.37 \mathrm{~cm} / \mathrm{s} ; n=249$ cycles) and during the low motion condition (gray; $<0.1 \mathrm{~cm} / \mathrm{s}$; mean motion, $0.04 \mathrm{~cm} / \mathrm{s} ; n=106$ cycles). Inset, Polar plot with vectors representing the angle and magnitude of the phase relationship for high motion (black) and low motion (gray) conditions. C, D, Summary plots of the average, absolute angular difference between phase preference vectors calculated from six MTCs ( $\boldsymbol{C}$, red bars) and $18 \mathrm{GCS}(\boldsymbol{D}$, blue bars) with statistically significant membrane potential phase modulation. ${ }^{* *} p<5 \times 10^{-5}$. The angular differences between the phase tuning during low and high respiratory frequencies regimens or in motion versus no motion conditions were statistically different from the mean angular differences calculated when the condition labels were randomized ( $p>0.05$ for both MTCs in C and GCs in D, $t$ test). phase coupling of the membrane potential $(p<0.01$ and $p<$ $10^{-4}$, paired $t$ test).

Finally, we assayed the difference in phase preference computed from different EPSP subgroups relative to the phase preference of the average membrane potential. Both the overall population and the large, fast EPSP subpopulation aligned closely with the membrane potential phase tuning (angular differences, $0.87 \pm 0.17$ and $0.85 \pm 0.16 \mathrm{rad}$, respectively; Fig. $6 C$ ), whereas response vectors computed from small, slow EPSPs were significantly more divergent from the membrane potential phase preference on average $(1.5 \pm 0.24 \mathrm{rad}, p<0.005, t$ test $)$. These results suggest that, although many different classes of EPSPs can contribute to the phase tuning in GCs, large-amplitude EPSPs with fast rise times (presumably proximal synaptic inputs subject to little cable filtering) can play a central role in regulating GC responses. The degree of alignment between the phase preference of the large, fast EPSPs and the membrane potential phase tuning was not correlated with the magnitude of the large, fast EPSP vector over a twofold variation in vector magnitude $\left(r^{2}=0.03\right.$; $p>0.05$; Fig. $6 D$ ). In contrast, the phase preference imparted by small, slow EPSPs (presumably primarily distal dendrodendritic EPSPs) came into alignment with the overall membrane potential phase tuning in GCs in rough proportion with the magnitude of small, slow EPSP response vector $\left(r^{2}=0.42 ; p<0.01\right.$; Fig. $\left.6 E\right)$.

\section{Discussion}

We make three principal conclusions in this study. First, we find that, under basal conditions (without experimentally controlled odor stimulation), the subthreshold synaptic input to both MTC and GC interneurons showed marked phase modulation (Fig. 2). We demonstrated phase modulation in MTCs and GCs using two independent methods: (1) direct averaging of respiratory cyclenormalized (Goldberg and Brown, 1969; Buonviso et al., 1992; Schusterman et al., 2011) membrane potentials; and (2) calculating the respiratory phases of individual EPSPs. Although previous groups have described phase modulation of MTC membrane potentials (Margrie and Schaefer, 2003; Fukunaga et al., 2012; Phillips et al., 2012), to our knowledge, this is the first quantitative report of phase modulation of subthreshold GC responses in awake mammals. The phase tuning of both principal cells and GC interneurons varied considerably between cells but was relatively constant within a recording session even when the respiratory frequency increased and during epochs of locomotion. Second, we find that phase modulation was often most pronounced within a relatively small subpopulation of EPSPs with large amplitudes and rapid kinetics-presumably synaptic inputs onto proximal dendritic segments that were subject to minimal cable attenuation. More frequent small-amplitude EPSPs with slow rise times, likely including many electrotonically remote dendrodendritic inputs, had less phase modulation (Figs. 5G, 6B) and often displayed phase preferences that differed from the average membrane potential (Fig. 6C). Finally, the ability of smallamplitude, slow EPSPs to influence the phase tuning of the overall membrane potential appeared to be dynamically modulated. Over the population of GCs analyzed, there was a strong and statistically significant correlation between the magnitude of the small, slow EPSP phase modulation and the correspondence between the phase preference imparted by this EPSP subpopulation and the phase tuning of the overall average membrane potential (Fig. 6E).

These results suggest a basal (nonstimulated) state model of the $\mathrm{OB}$ in which interneurons and principal cells maintain a timing signal that is coupled to the respiratory rhythm. The variety of 
A
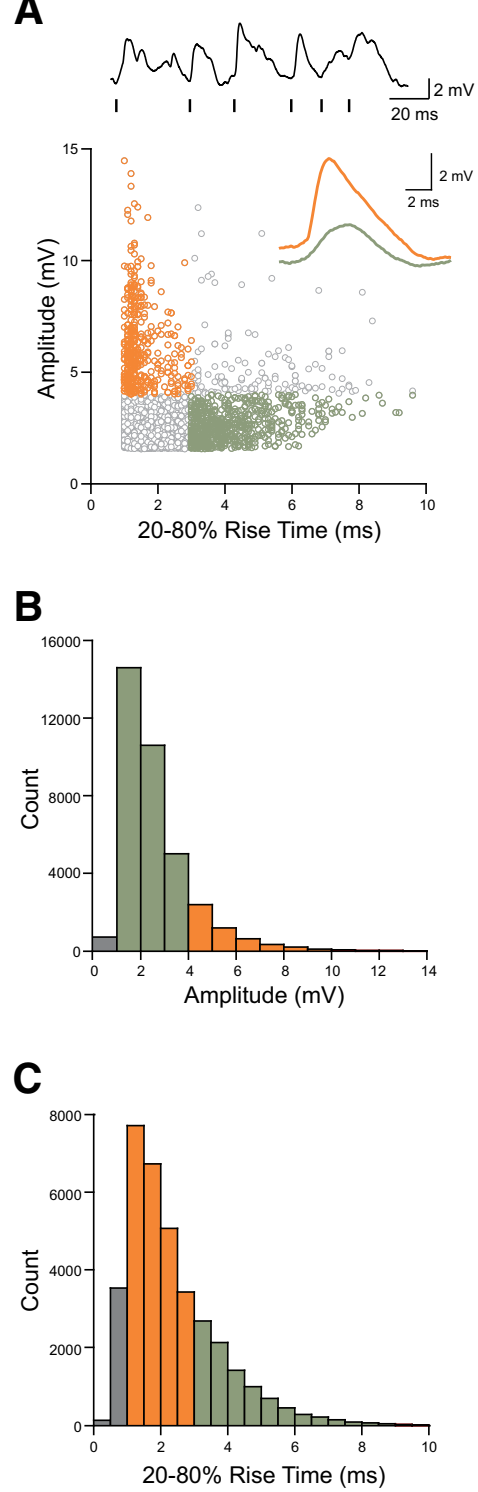

D

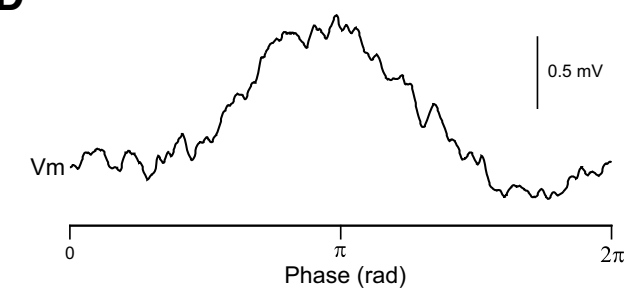

E
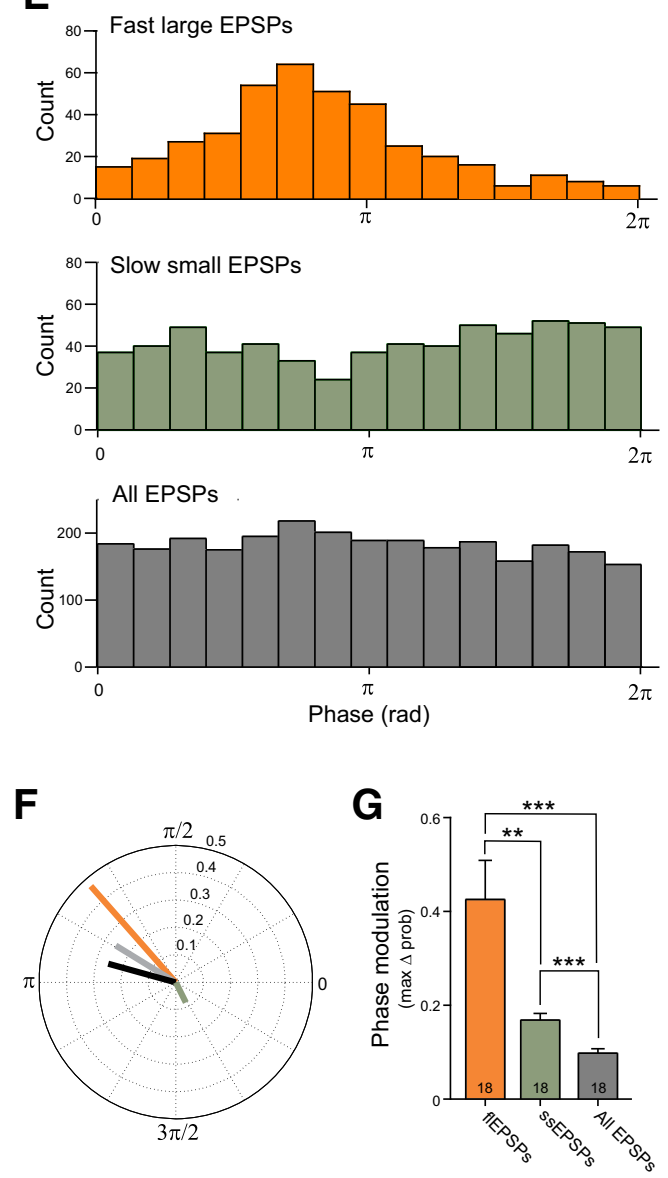

Figure 5. Phase modulation of spontaneous EPSPs in GCS. $\boldsymbol{A}$, Scatter plot of the rise times and amplitudes of EPSPs of a representative GC. Two subpopulations of EPSPs were considered: (1) fast, large EPSPS (fIEPSP; orange symbols: rise time between 1 and $3 \mathrm{~ms}$, amplitude between 4 and $15 \mathrm{mV}$ ); and (2) slow, small EPSPs (ssEPSPs; green symbols; rise time between 3 and $10 \mathrm{~ms}$, amplitude between 1 and $4 \mathrm{mV}$ ). Gray points represent EPSPs that were not part of either the fIEPSP or sSEPSP subpopulations. Sample membrane potential trace with vertical lines indicating detected EPSPs (top) and examples of a fast, large EPSP (orange) and a slow, small EPSP (green) from the same GC (bottom) shown in the inset. $\boldsymbol{B}, \boldsymbol{C}$, Distributions of EPSP amplitudes $(\boldsymbol{B})$ and rise times $(\boldsymbol{C})$ for the $18 \mathrm{GCS}$ with statistically significant average membrane potential phase modulation. Histogram bars colored to match symbol colors in $A$ (and continued throughout figure). $\boldsymbol{D}$, The mean, phase-aligned membrane potential from the same GC shown in $\boldsymbol{A}$ (peak phase preference, 2.9 rad). $\boldsymbol{E}$, Histograms of the phase position of all EPSPs (gray bars) and both the fIEPSP (orange bars) and ssEPSP (green bars) subpopulations. In this GC, fast, large EPSPs have an obvious phase preference peak that occurs slightly before the peak depolarization of the mean, phase aligned membrane potential in $\boldsymbol{D}$ (at $2.25 \mathrm{rad} ; n=400 \mathrm{EPSPs}$ comprising $14.4 \%$ of all EPSPs in this GC; orange). In contrast, the timing of ssEPSPs ( $n=630$ EPSPs; $22.7 \%$ of EPSPs; green) in the same neuron exhibited muchless phase modulation. $F$, Polar plot with vectors depicting the angle and magnitude of the phase relationship for the mean membrane potential (black), all EPSPs (gray), fast, large EPSPs (orange), and slow, small EPSPs (green) in the same GC. G, Over the population of $18 \mathrm{GCS}$ with membrane potential phase modulation, the timing of flEPSPs (orange bar) had significantly more phase modulation than either the ssEPSP subpopulation (green; ${ }^{* *} p<$ 0.005 ) or the complete set of all EPSPs (gray; ${ }^{* * *} p<5 \times 10^{-4}$ ). The timing of ssEPSPs had slighter more phase modulation across the same $18 \mathrm{GCs}$ than the complete set of all EPSPs $\left(4.0 \%\right.$ increase; $\left.{ }^{* * *} p<5 \times 10^{-4}\right)$.

respiratory phase preferences we observed in the subthreshold activity of $\mathrm{OB}$ neurons likely reflects coordination of distinct local circuits based on their phase tuning. Because phase preference was not uniform across GCs, phase modulation of the intracellular voltage likely introduces a temporal bias that helps define which subgroups of GCs will respond to a sensory input. The prominent role we find for large-amplitude EPSPs with fast kinetics in setting the phase preference of GCs suggests that a critical timing signal may arise from cortical feedback projections. Given sufficiently large phase modulation in their timing (perhaps during odor responses that trigger robust spiking in phase-related MTCs), slow, smallamplitude EPSPs also appear to play a larger role in governing the phase preference of GCs. Therefore, interneuron respiratory phase preference may be regulated primarily by cortical feedback (and other potential sources of proximal excitatory synaptic input) during basal conditions but shift to reflect the phase tuning of active presynaptic principal (mitral/tufted) cells during sensory stimulation. Such a dual-pathway phase modulation mechanism would promote variation among GC phase tuning but still enable large populations of GCs to be recruited (and phase synchronized) in response to strong sensory input. Given their potential to influence somatic APs, the timing of large-amplitude inputs could also serve to modulate plasticity at dendritic synapses.

In this study, we maintained the intracellular membrane potential near $-70 \mathrm{mV}$ to facilitate comparisons across different neurons and to minimize the contribution of fast inhibitory synaptic potentials to phasealigned average membrane potential. By focusing on subthreshold EPSPs, our results do not directly address the relationship between EPSP phase timing and spike timing in GCs, but this is obviously a critical question given the ability of backpropagating APs to regulate distal dendrodendritic microcircuits (Isaacson and Strowbridge, 1998; Balu et al., 2007). The phase advance we find for spontaneous EPSPs relative to the phase tuning of the average membrane potential is consistent with a model of membrane potential modulation reflecting EPSP temporal summation. This model predicts that GC spiking probability will follow the depolarizing peak in the average membrane potential, often reflecting the timing of fast, large-amplitude EPSPs during the basal (unstimulated) state. Another prediction from this study is that, during odor stimulation, the timing of GC spike activity will shift to reflect a larger contribution of phase preferences imparted by distal, dendrodendritic EPSPs as presynaptic mitral and tufted neurons are activated. However, assaying odor-stimulated activity in GCs is technically challenging given the sparse nature of odor coding in these interneurons (Cazakoff et al., 2014) and the limited duration intracellular recordings are feasible in awake, head-fixed mice. This question is likely to 
be more easily addressed in future studies using optogenetic stimulation methods to directly activate large subpopulations of olfactory sensory afferents to the OB.

Previous work has suggested that the output of the OB may be composed of separate, functional channels (Fukunaga et al., 2012; Haddad et al., 2013). The activity of principal cells can be approximately segregated into groups that have peaks of activity early versus late in the respiratory cycle, which correspond morphologically to mitral and tufted cells, respectively (Fukunaga et al., 2012). Anterograde axonal tracing and antidromic stimulation of MTCs also supports the theory of MTC activity being functionally distinct because MTCs can have predominantly non-overlapping projection target regions (Schneider and Scott, 1983; Igarashi et al., 2012). The projection targets of OB output typically also send feedback projections back to the $\mathrm{OB}$ (Luskin and Price, 1983). It is not known whether cortical neurons that generate feedback projections to the OB complement MTC projections with cell type (or region)specific respiratory coupling patterns. The heterogeneity in the phase preferences of potential presynaptic $\mathrm{OB}$ and olfactory cortical neurons likely underlies the variability we observed in GC EPSP and membrane potential phase tuning. GCs can be subdivided into classes that receive orthodromic excitatory input primarily from mitral or tufted cells [based on the laminar distribution GC bodies within the GCL and dendritic arbors within the external plexiform layer (EPL); Orona et al., 1983]. Because our recordings were sampled from a broad range of depths within the GCL, our results likely included both GCs excited primarily by mitral cells and GCs driven by tufted cells. This variability between GC subclasses also likely contributes to the mixture of GC phase preferences we observed, especially within the phase tuning generated by smallamplitude EPSPs with slow kinetics (presumably mostly distal, dendrodendritic inputs; Balu et al., 2007).

Although we find that phase tuning of large-amplitude EPSPs with fast rise times often was aligned closely with phase preference calculated from the average membrane potential, our recording conditions (with neurons held near $-70 \mathrm{mV}$ ) precluded directly assaying the effectiveness of different EPSP subclasses in triggering phase-aligned spiking in GCs. Proximal EPSPs may trigger APs more effectively because of their large amplitude, although GCs receive more frequent small-amplitude EPSPs, which could summate to different spiking, especially during sensory stimulation. This question could be addressed in future studies using spike-triggered averaging with GCs held at more depolarized potentials. The surprising reduction in the respiratory modulation of GC spiking dur-

B
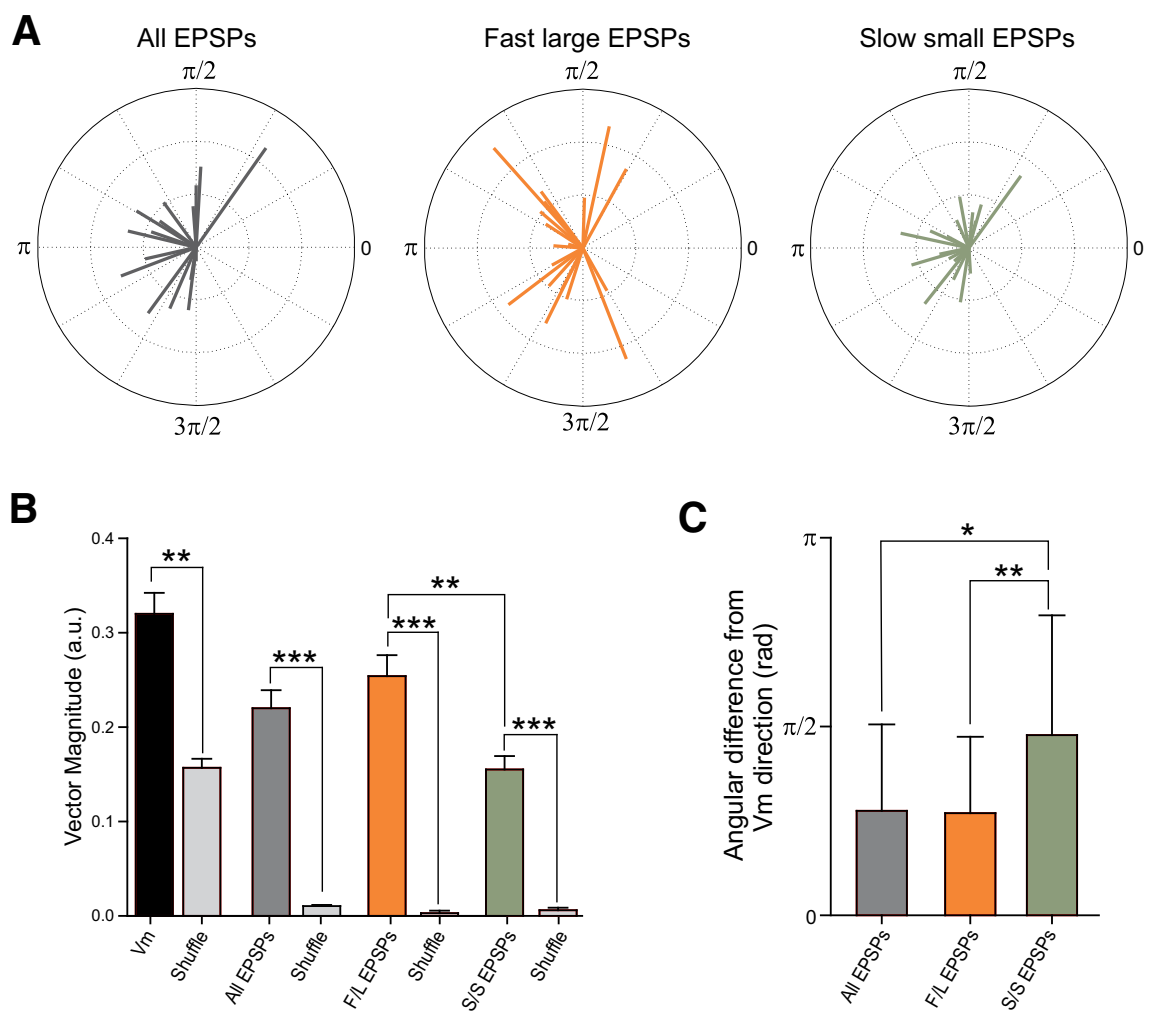

C

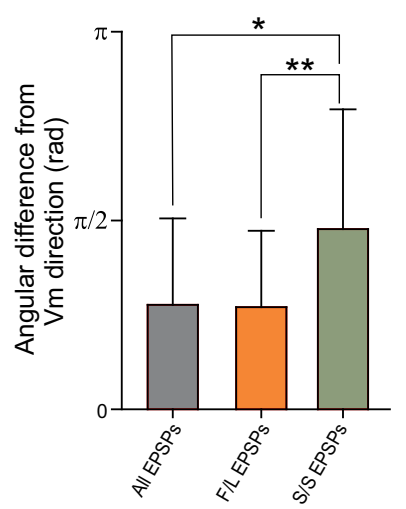

$\mathbf{E}$
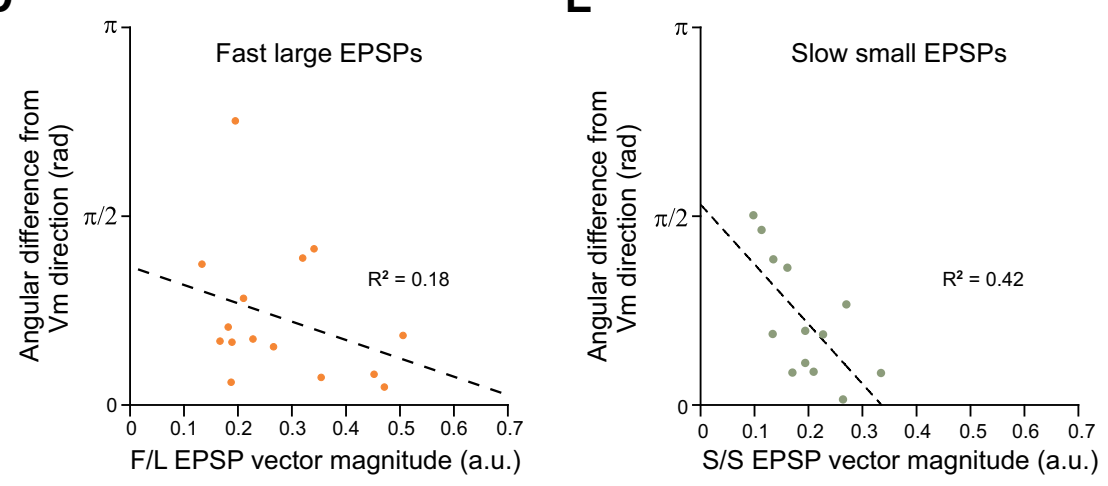

Figure 6. Phase tuning of fast, large-amplitude EPSPs resembles the phase modulation of the average membrane potential. $\boldsymbol{A}$, Polar plots of the vector magnitude by the vector angle for all $18 \mathrm{GCs}$ with significant membrane potential respiratory coupling. Vectors calculated from the average membrane potential in each GC of all EPSP (gray, left), fast, large EPSP (F/L EPSPs; orange, middle), and slow, Small EPSP (S/SEPSPs; green, right) subpopulations. $\boldsymbol{B}$, Plot of the mean vector magnitudes calculated the mean membrane potential (black), from all EPSPs (dark gray), from F/L EPSPs (orange), and from S/S EPSPs (green). ${ }^{* *} p<0.005$, ${ }^{* * *} p<10^{-7}$. C, The average absolute angular difference between the membrane potential vector and the vector calculated from different group of EPSPS. All EPSPs (gray) and F/L EPSPs (red) are both significantly closer to the membrane potential vector than S/S EPSPS $\left({ }^{*} p<0.05\right.$ and ${ }^{* *} p<0.005$, respectively). $\boldsymbol{D}$, Plot of absolute angular difference between the membrane potential vector and the F/L EPSP vector magnitude for GCS with F/L EPSP vector distributions significantly different from uniform distributions ( $p<0.05$, Rayleigh's test, $n=15$ of $18 \mathrm{GCs})$. A dashed line shows a trend in the data that are not significant $\left(r^{2}=0.030\right.$, $p=0.25)$. $E$, Plot of the absolute angular difference between the membrane potential vector and the S/SEPSP vector magnitude, including only cells with S/S EPSP vector distributions significantly different from uniform distributions ( $p<0.05$, Rayleigh's test, $n=13$ of $18 \mathrm{GCS}$ ). A dashed line illustrates a significant, negative correlation between S/S EPSP vector magnitudes and the difference between the angle of the S/S EPSP vector and the membrane potential vector $\left(r^{2}=0.42, p<0.01\right)$.

ing shifts from the anesthetized to awake state (Cazakoff et al., 2014) raises the possibility that summation of slow dendrodendritic EPSPs can be more effective at triggering APs under some conditions. Alternatively, the modest degree of spike phase modulation observed during the awake state may reflect the larger diversity of presynaptic inputs to GCs that are active during wakefulness (Bloms-Funke et al., 1999). This hypothesis is consistent with the higher mean GC spike rate observed during the awake versus anesthetized state (Ca- 
zakoff et al., 2014). During wakefulness, GC spiking is likely driven by a more diverse set of excitatory afferents, eliciting spikes at different phases relative to the respiratory cycle. The functional significance of many "out-of-phase" EPSPs (e.g., small-amplitude EPSPs that impart a phase tuning that is distinct from the average membrane potential tuning) we observed remains to be explored. It is possible that these synaptic inputs provide each GC with a canvas of "alternate phase tunings" that are selected dynamically by cortical inputs or through summation with orthodromic input during sensory stimulation. If individual GCs are able to modulate their membrane potential phase tuning, this may provide a mechanism to select among different subgroups of presynaptic MTCs that respond to sensory input with different phase preferences.

\section{References}

Abraham NM, Egger V, Shimshek DR, Renden R, Fukunaga I, Sprengel R, Seeburg PH, Klugmann M, Margrie TW, Schaefer AT, Kuner T (2010) Synaptic inhibition in the olfactory bulb accelerates odor discrimination in mice. Neuron 65:399-411. CrossRef Medline

Balu R, Pressler RT, Strowbridge BW (2007) Multiple modes of synaptic excitation of olfactory bulb granule cells. J Neurosci 27:5621-5632. CrossRef Medline

Berens P (2009) CircStat: a MATLAB toolbox for circular statistics. J Stat Softw 31:1-21.

Blanton MG, Lo Turco JJ, Kriegstein AR (1989) Whole cell recording from neurons in slices of reptilian and mammalian cerebral cortex. J Neurosci Methods 30:203-210. CrossRef Medline

Bloms-Funke P, Gernert M, Ebert U, Löscher W (1999) Extracellular singleunit recordings of piriform cortex neurons in rats: Influence of different types of anesthesia and characterization of neurons by pharmacological manipulation of serotonin receptors. J Neurosci Res 55:608-619. CrossRef Medline

Boyd AM, Sturgill JF, Poo C, Isaacson JS (2012) Cortical feedback control of olfactory bulb circuits. Neuron 76:1161-1174. CrossRef Medline

Buonviso N, Chaput MA, Berthommier F (1992) Temporal pattern analyses in pairs of neighboring mitral cells. J Neurophysiol 68:417-424. Medline

Carey RM, Wachowiak M (2011) Effect of sniffing on the temporal structure of mitral/tufted cell output from the olfactory bulb. J Neurosci 31: 10615-10626. CrossRef Medline

Cazakoff BN, Lau BYB, Crump KL, Demmer HS, Shea SD (2014) Broadly tuned and respiration-independent inhibition in the olfactory bulb of awake mice. Nat Neurosci 17:569-576. CrossRef Medline

Cenier T, McGann JP, Tsuno Y, Verhagen JV, Wachowiak M (2013) Testing the sorption hypothesis in olfaction: a limited role for sniff strength in shaping primary odor representations during behavior. J Neurosci 33:79-92. CrossRef Medline

Chen WR, Shepherd GM (1997) Membrane and synaptic properties of mitral cells in slices of rat olfactory bulb. Brain Res 745:189-196. CrossRef Medline

Fukunaga I, Berning M, Kollo M, Schmaltz A, Schaefer AT (2012) Two distinct channels of olfactory bulb output. Neuron 75:320-329. CrossRef Medline

Gire DH, Whitesell JD, Doucette W, Restrepo D (2013) Information for decision-making and stimulus identification is multiplexed in sensory cortex. Nat Neurosci 16:991-993. CrossRef Medline

Goldberg JM, Brown PB (1969) Response of binaural neurons of dog superior olivary complex to dichotic tonal stimuli: some physiological mechanisms of sound localization. J Neurophysiol 32:613-636. Medline

Grosmaitre X, Santarelli LC, Tan J, Luo M, Ma M (2007) Dual functions of mammalian olfactory sensory neurons as odor detectors and mechanical sensors. Nat Neurosci 10:348-354. CrossRef Medline

Haddad R, Lanjuin A, Madisen L, Zeng H, Murthy VN, Uchida N (2013) Olfactory cortical neurons read out a relative time code in the olfactory bulb. Nat Neurosci 16:949-957. CrossRef Medline

Hyde RA, Strowbridge BW (2012) Mnemonic representations of transient stimuli and temporal sequences in the rodent hippocampus in vitro. Nat Neurosci 15:1430-1438. CrossRef Medline

Igarashi KM, Ieki N, An M, Yamaguchi Y, Nagayama S, Kobayakawa K, Kobayakawa R, Tanifuji M, Sakano H, Chen WR, Mori K (2012) Parallel mitral and tufted cell pathways route distinct odor information to different targets in the olfactory cortex. J Neurosci 32:7970-7985. CrossRef Medline

Isaacson JS, Strowbridge BW (1998) Olfactory reciprocal synapses: dendritic signaling in the CNS. Neuron 20:749-761. CrossRef Medline

Lowe G (2002) Inhibition of backpropagating action potentials in mitral cell secondary dendrites. J Neurophysiol 88:64-85. Medline

Luskin MB, Price JL (1983) The topographic organization of associational fibers of the olfactory system in the rat, including centrifugal fibers to the olfactory bulb. J Comp Neurol 216:264-291. CrossRef Medline

Margrie TW, Schaefer AT (2003) Theta oscillation coupled spike latencies yield computational vigour in a mammalian sensory system. J Physiol 546:363-374. CrossRef Medline

Margrie TW, Sakmann B, Urban NN (2001) Action potential propagation in mitral cell lateral dendrites is decremental and controls recurrent and lateral inhibition in the mammalian olfactory bulb. Proc Natl Acad Sci U S A 98:319-324. CrossRef Medline

Onoda N, Mori K (1980) Depth distribution of temporal firing patterns in olfactory bulb related to air-intake cycles. J Neurophysiol 44:29-39. Medline

Orona E, Scott JW, Rainer EC (1983) Different granule cell populations innervate superficial and deep regions of the external plexiform layer in rat olfactory bulb. J Comp Neurol 217:227-237. CrossRef Medline

Phillips ME, Sachdev RN, Willhite DC, Shepherd GM (2012) Respiration drives network activity and modulates synaptic and circuit processing of lateral inhibition in the olfactory bulb. J Neurosci 32:85-98. CrossRef Medline

Pressler RT, Strowbridge BW (2006) Blanes cells mediate persistent feedforward inhibition onto granule cells in the olfactory bulb. Neuron 49:889 904. CrossRef Medline

Pressler RT, Inoue T, Strowbridge BW (2007) Muscarinic receptor activation modulates granule cell excitability and potentiates inhibition onto mitral cells in the rat olfactory bulb. J Neurosci 27:10969-10981. CrossRef Medline

Pressler RT, Rozman PA, Strowbridge BW (2013) Voltage-dependent intrinsic bursting in olfactory bulb Golgi cells. Learn Mem 20:459-466. CrossRef Medline

Rubin BD, Katz LC (1999) Optical imaging of odorant representations in the mammalian olfactory bulb. Neuron 23:499-511. CrossRef Medline

Schmidt LJ, Strowbridge BW (2014) Modulation of olfactory bulb network activity by serotonin: synchronous inhibition of mitral cells by spatially localized GABAergic microcircuits. Learn Mem 21:406-416. CrossRef Medline

Schneider SP, Scott JW (1983) Orthodromic response properties of rat olfactory bulb mitral and tufted cells correlate with their projection patterns. J Neurophysiol 50:358-378. Medline

Shusterman R, Smear MC, Koulakov AA, Rinberg D (2011) Precise olfactory responses tile the sniff cycle. Nat Neurosci 14:1039-1044. CrossRef Medline

Wachowiak M, Cohen LB (2001) Representation of odorants by receptor neuron input to the mouse olfactory bulb. Neuron 32:723-735. CrossRef Medline

Youngstrom IA, Strowbridge BW (2012) Visual landmarks facilitate rodent spatial navigation in virtual reality environments. Learn Mem 19:84-90. CrossRef Medline 